FORMATLON Formation emploi

Revue française de sciences sociales

132 | octobre-décembre 2015

Enseignement supérieur et mondes économiques : de nouveaux éclairages

\title{
Postface : De la mise en concurrence à la mise en marché de l'enseignement supérieur
}

Hugo Harari-Kermadec et Léonard Moulin

\section{(2) OpenEdition}

Journals

Édition électronique

URL : http://journals.openedition.org/formationemploi/4590

DOI : 10.4000/formationemploi.4590

ISSN : 2107-0946

Éditeur

La Documentation française

Édition imprimée

Date de publication : 30 décembre 2015

Pagination : $91-103$

ISSN : 0759-6340

Référence électronique

Hugo Harari-Kermadec et Léonard Moulin, « Postface : De la mise en concurrence à la mise en marché de l'enseignement supérieur », Formation emploi [En ligne], 132 | octobre-décembre 2015, mis en ligne le 28 janvier 2016, consulté le 30 octobre 2020. URL : http://journals.openedition.org/

formationemploi/4590 ; DOI : https://doi.org/10.4000/formationemploi.4590

(c) Tous droits réservés 


\title{
Postface
}

\section{De la mise en concurrence à la mise en marché de l'enseignement supérieur}

\author{
Hugo Harari-Kermadec \\ Maître de conférences à l'École Normale Supérieure de Cachan, Institutions et Dynamiques \\ Historiques de l'Économie et de la Société, CNRS UMR 8533.
}

\begin{abstract}
LÉONARD MOULIN
Attaché Temporaire d'Enseignement et de Recherche, université Paris 13, Centre d'Économie de I'université Paris Nord, CNRS UMR 7234.
\end{abstract}

L'enseignement supérieur fait l'objet d'une succession de réformes et de transformations dans un cadre néolibéral, à partir des années 1980, qui ont renforcé les liens et les similarités avec le monde économique.

Les deux articles historiques de ce dossier nous rappellent que ces liens n'ont pas commencé avec le processus de Bologne (1999) et la stratégie de Lisbonne (2000). Christelle DormoyRajramanan et Antoine Derouet nous sortent en effet de notre contexte de crise et de chômage de masse. Ils évoquent les Trente Glorieuses, lorsque le système universitaire est invité à fournir la main-d'œuvre formée nécessaire pour accompagner la croissance économique, et que les écoles d'ingénieurs s'interrogent sur leurs formations, et surtout sur leurs enseignants, qui feront des ingénieurs des cadres capables d'appréhender les hommes autant que la technologie. Si le discours porte déjà sur la nécessaire mise en conformité de l'université avec les besoins de l'activité économique, ce rôle de préparation à l'entrée dans le monde économique laisse un espace propre à l'enseignement supérieur, en amont et donc dans une certaine extériorité : c'est bien parce que le monde économique n’assure pas lui-même la formation, que ce soit par des établissements d'enseignement supérieur privés ou en interne après le recrutement, qu'il a besoin d'un système public d'enseignement supérieur.

Les liens sont donc anciens, mais la période récente marque-t-elle une rupture ? Les articles de Simon Paye et Aurélien Casta s'intéressent à deux incursions plus directes du monde économique dans l'enseignement supérieur : la pénétration, au Royaume-Uni, des méthodes de gestion des ressources humaines issues du privé, souvent par le recrutement de managers qui en proviennent, pour le premier ; par le développement des établissements d'enseignement supérieurs privés, pour le second.

Finalement, l'objet de chacun des quatre textes de ce dossier peut servir de point de comparaison éclairant sur le système d'enseignement supérieur public français contem- 
porain, qui n'est plus celui du vingtième siècle, sans être (encore ?) dominé par les établissements privés ni géré comme eux.

C'est particulièrement précieux dans la mesure où cette prise de recul permet de replacer la situation nationale contemporaine dans une dynamique historique et un contexte international. On peut alors reprendre la discussion ouverte par Olivia Chambard et Laurène Le Cozanet en introduction à propos de la " marchandisation » ou l' "économicisation " de l'enseignement supérieur.

Comment donner un sens commun à un ensemble de transformations récentes et en cours, sans tomber dans la réification d'une université imaginaire ? Nous proposons de les envisager comme participant d'une " mise en marché» (Jongbloed, 2003), une évolution de l'enseignement supérieur vers le fonctionnement du monde économique. Pour ce faire, nous nous appuierons sur deux transformations, intimement liées : l'évolution et le renforcement de la concurrence entre établissements, d'une part, et la hausse des frais d'inscription, d'autre part.

\section{Classements et concurrence entre établissements}

À l'époque néolibérale, la concurrence est une des caractéristiques attendues de l'économie, et son insuffisance est considérée comme un symptôme inquiétant. On peut alors appréhender l'évolution des systèmes d'enseignement supérieur vers un fonctionnement économique à travers l'extension de la concurrence en leur sein, ou, pour être plus complet, de leur " mise en marché». Selon Jongbloed (2003, p. 113), il s'agit des évolutions qui "visent à renforcer le choix des étudiants et liberaliser les marchés afin d'augmenter la qualité et la variété des services offerts par les fournisseurs d'enseignement supérieur ".

Jongbloed (2003) spécifie ainsi huit critères permettant de mesurer la mise en marché de l'enseignement supérieur : pour les universités, la liberté d'entrer sur le marché, de spécifier leur produit, d'utiliser les ressources disponibles, de déterminer les prix, et pour les consommateurs, la liberté de choisir le fournisseur, le produit, de disposer d'information sur les prix et la qualité et sur la capacité des prix à couvrir les coûts des études.

La définition de ces huit conditions permet de comprendre comment différents pays ont progressivement introduit des mécanismes de marché dans leur système d'enseignement supérieur. La France, par exemple, s'apparente sans doute davantage à un "quasi-marché»1 qu’à un marché, dans la mesure où son système d'enseignement supérieur intègre progressivement des mécanismes de marché, sans pour autant les adopter tous.

1. Pour reprendre le terme utilisé par Teixeira et al. (2004). 
Les pays en retard sont néanmoins poussés dans le sens du mouvement par un renouvellement de l'internationalisation de l'enseignement supérieur. Les années 2000 marquent en effet un tournant international dans la mise en concurrence des établissements.

Si la recherche est, depuis très longtemps, internationalisée - avec des différences entre disciplines - l'espace de l'enseignement supérieur était resté, quant à lui, principalement national.

C'est d'ailleurs essentiellement à partir de la recherche que sont construits les classements internationaux qui instituent autant qu'ils rendent compte de cette concurrence (Espeland et Sauder, 2007). Pour Granger (2015), les universités sont ainsi enjointes à rattraper leur "retard relatif".

Par leur format, les classements comme l'Academic Ranking of World Universities, dit "classement de Shanghai », font en effet émerger une catégorie d'établissements " de classe mondiale $»^{2}$ qui ne préexistait pas à leur mise en ordre. Cette catégorie ne contient qu'une faible partie des établissements (on trouve par exemple 33 établissements français sur le site du classement de Shanghai, sur les 142 régis par le Code de l'éducation ${ }^{3}$. Conforme à son objet, ce classement académique des universités mondiales annonce sur son site ${ }^{4}$ s'intéresser à 1200 universités avant de publier le rang des 500 meilleures.

Les regroupements d'établissements promus par une série d'initiatives réglementaires et d'incitations budgétaires (pôles régionaux, Idex, Comue, etc.), dans les années 2000, restructurent les établissements suivant une échelle mieux adaptée au classement : il s'agit d'obtenir une "visibilité internationale ". S’il est caricatural de penser le classement de Shanghai comme élément moteur de ces regroupements, il aide à envisager un paysage universitaire français inséré dans une compétition internationale et à distinguer les établissements qui y participent de ceux qui en sont exclus.

Les classements s'inscrivent en effet dans les discours des responsables politiques et des chefs d'établissements comme un environnement avec lequel il faudrait compter, qu'on les juge pertinents ou non.

On peut néanmoins estimer qu'il s'agit davantage d'éléments de justification de réformes préétablies (Gingras, 2013, p. 105 ; Barats et Leblanc, 2012). Un des effets pervers d'une adaptation à cette internationalisation par les classements est qu'elle produit (peut-être) des établissements d'excellence mais certainement pas des systèmes nationaux d'excellence (Hazelkorn, 2015). C'est en tout cas la crainte d'une partie des membres de la Conférence des Présidents d'Universités (CPU) françaises, conduisant au dépôt de deux listes concurrentes pour présider la CPU. Les présidents de 21

\footnotetext{
2. Pour reprendre le terme emprunté à la conférence (13 mai 2015) éponyme "Vers de nouvelles universités de classe mondiale », organisée par Paris Sciences et Lettres.

3. Titre Ier du livre 7 du code de l'éducation.

4. http://www.shanghairanking.com/fr/.
} 
universités exclues des classements internationaux signent ainsi, le 29 mai 2015, une tribune collective ${ }^{5}$ intitulée «Quel avenir pour l'enseignement supérieur et la recherche français ?". Ils y dénoncent cette évolution : "L'obsession des classements internationaux prévaut dans les choix de soutien budgétaire, la notion d'excellence scientifique et de masse critique deviennent centrales, au détriment des autres missions de l'université, dont la réussite pour tous ou la formation tout au long de la vie."

Ainsi, les moyens se concentrent dans quelques établissements compétitifs, au détriment de nombreux autres, qui prennent pourtant en charge l'essentiel de la formation supérieure de la population. Ainsi, selon Gardner (2008), en cherchant à faire gagner des places aux universités australiennes qui se hissent dans le haut des classements internationaux, on met de côté $94 \%$ des étudiants. On retrouve une stratégie similaire à celle des "champions " nationaux (ou européens) dans les secteurs de l'économie marchande mondialisée, comme l'aéronautique ou l'énergie.

L'internationalisation de l'enseignement supérieur ne se limite pas à une comparaison purement discursive des établissements d'un pays à l'autre. Parallèlement au développement du nombre et de la portée médiatique des classements, on assiste à l'essor du nombre d'étudiants en mobilité internationale - passé de deux à quatre millions entre 2000 et $2012^{6}$. C'est donc aussi à cette échelle que les étudiants peuvent choisir leur "fournisseur", pour reprendre les termes de Jongbloed (2003).

C'est bien dans cette logique de mise en marché qu'il faut lire cette augmentation du nombre d'étudiants en mobilité internationale : si l'accueil d'étudiants étrangers a pu participer d'une politique de rayonnement culturel et économique, désormais, ils "représentent une manne pour les pays d'accueil" (OCDE, 2013a, p. 4). Le Canada, qui occupe la sixième place dans le classement des pays accueillant le plus d'étudiants en mobilité, estime à huit milliards de dollars canadiens (environ 5,6 milliard d'euros) les dépenses de ces étudiants sur son sol, en 2010.

Si ces montants incluent des dépenses courantes et de logement, les frais d'inscription des étudiants en mobilité constituent une source de revenu significative pour certains établissements. La plupart des pays de l'OCDE (Organisation de coopération et de développement économiques) autorisent d'ailleurs leurs établissements à facturer des frais plus élevés pour les étudiants étrangers - une perspective à l'étude en France selon Genevière Fioraso ${ }^{7}$, alors secrétaire d'Etat à l'Enseignement supérieur et la Recherche.

5. Article publié sur le site de Médiapart, le 29 mai 2015.

6. Chiffres de l'UNESCO Institute of Statistics.

7. Voir l'article d'Isabelle Rey-Lefebvre et de Nathalie Brafman, du 26 novembre 2014, intitulé «Etudiants étrangers : une ressource pour financer l'université ? ", Le Monde. 


\section{De la concurrence à la mise en marché}

Pour que la mise en concurrence soit une mise en marché, il faut, selon les critères de Jongbloed (2003), des prix couvrant l'essentiel des coûts. C'est notamment le cas dans de nombreux pays ayant procédé récemment à une augmentation des frais d'inscription, tels que l'Australie, l'Angleterre, les États-Unis, la Nouvelle-Zélande ou les Pays-Bas (OCDE, 2013b), ou ceux qui ont appliqué de telles augmentations, au moins pour les étudiants étrangers, comme la Suède, ou qui ont affiché leur volonté de le faire mais se sont retrouvés contrariés par des mouvements étudiants, comme ce fut le cas au Québec ou plus récemment en Afrique du sud.

En France, si les diplômes universitaires nationaux sont soumis à une tarification unique et modérée, les appels à son augmentation ne manquent pas ces dernières années, de même que la multiplication des statuts dérogatoires accordés à certains établissements, tels que l'université Paris 9 Dauphine, les Écoles des Mines et des Télécoms ou Sciences Po Paris. Visant les étudiants étrangers, ceux des classes préparatoires aux grandes écoles ou ceux de masters, l'augmentation des frais d'inscription est mise en avant par des Think Tank comme Terra Nova (Lichtenberger, Aïdara, 2011), l'Institut de l'entreprise (Chiapori, 2011) ou l'Institut Montaigne (Colin, 2008), de nombreux universitaires ou femmes et hommes politiques (ACIDES, 2015).

En 2012, la CPU a par ailleurs chargé l'Insee d'évaluer l'impact d'un quadruplement des frais d'inscription dans les universités françaises et plaide pour une hausse de ceux-ci. Les simulations de l'Insee (2012) reposent sur une augmentation des frais d'inscription et la suppression de la demi-part fiscale, profitant aux ménages les plus aisés $^{8}$, au profit de sa réallocation sous forme d'aides (forfaitaires ou progressives). L'Insee (2012) en conclut qu'une telle politique accroîtrait la redistributivité et donc l'équité du financement du système d'enseignement supérieur. De la même manière, une récente étude de France Stratégie (Charles et Delpech, 2015) proposait de porter les frais d'inscription des étudiants étrangers à 6000 euros en licence et à 12000 euros en master (contre respectivement 183 et 254 euros aujourd'hui). Plus récemment, une étude des ministères de l'Économie, des Finances et de l'Industrie et de l'Éducation nationale, de l'Enseignement supérieur et de la Recherche (Hautier et al., 2015) proposait une augmentation des frais d'inscription à 1000 euros par an.

Au niveau académique, la mise en marché de l'enseignement supérieur peut s'appuyer sur la théorie du capital humain. Cette théorie, qui vise précisément à analyser l'éducation, en

8. Les avantages fiscaux des plus aisés (majoration du quotient familial pour les étudiants rattachés au foyer fiscal de leurs parents et réduction d'impôt pour frais de scolarité des enfants poursuivant des études supérieures) représentent, selon nos calculs à partir des chiffres de l'année 2013 fournis par le MESR (2015), $77 \%$ du montant total des bourses dont bénéficient les ménages les plus modestes. 
général et l'enseignement supérieur, en particulier dans l'optique de la rendre efficace pour le monde économique, remonte notamment aux travaux de Becker (1962). Pour ce dernier, il existe en effet des "activités qui modifient le revenu réel futur à travers l'incorporation de ressources dans les individus" (Becker, 1962, p. 9). Selon Gazier (1992, p. 193), ces activités déterminent ainsi "l'ensemble des capacités productives d'un individu (ou d'un groupe), incluant ses aptitudes opératoires au sens le plus large: connaissances générales ou spécifiques, savoir-faire, expérience... ", i.e. son "capital humain ", et dont la valeur n'est déterminée que sur le marché du travail.

L'enseignement supérieur, en raison des rendements privés importants qu'il procure?', que les économistes ont a de nombreuses reprises tenté d'estimer (voir notamment Card, 1999 ; Harmon et al., 2003 ; Hendricks, 2004), constitue l'exemple par excellence d'investissement en capital humain.

Conformément aux préceptes de la théorie économique néoclassique, les étudiants, supposés disposer d'une information parfaite, décident d'étudier si les gains (rendements) attendus associés à la poursuite d'études sont supérieurs aux coûts qu'elle engendre. Cette théorie attribue ainsi une valeur marchande à l'éducation dans la mesure où celle-ci y est décrite comme un moyen d'accroître la productivité des étudiants, et donc comme un investissement productif. Considérer l'éducation au prisme de cette théorie revient à considérer l'enseignement non plus comme l'émancipation intellectuelle du plus grand nombre mais avant tout comme une activité économique qui se résume à des relations entre clients et fournisseurs (Rotschild et White, 1995).

Dans cette perspective, les frais d'inscription apparaissent alors comme les indicateurs justes et naturels du capital humain que permet d'acquérir une formation, comme la promesse d'un rendement futur qui donne son sens à l'inscription.

A partir de ce cadre, Gary-Bobo et Trannoy $(2005$; 2008) ont développé une argumentation en faveur de l'augmentation des frais d'inscription en France. Cette argumentation se centrait sur l'efficience des modes de sélection à l'entrée de l'université, en discutant notamment de la substituabilité entre examens d'entrée et frais d'inscription. Ces auteurs en déduisaient, sous réserve d'un accès parfait au crédit (et donc de la construction d'un mécanisme ad hoc), que les frais d'inscription se révèleraient socialement optimaux dans la mesure où ils pourraient conduire les étudiants à s'autosélectionner en fonction de leur talent.

9. Ce cadre analytique sous-estime cependant les externalités positives de l'éducation, dont on sait pourtant qu'elles sont importantes (Grossman, 2006), en particulier en termes de criminalité (Garces et al. 2002 ; Lochner et Moretti, 2004), de santé (Grossman et Kaestner 1997), de croissance économique (Aghion et Howitt, 2009), de perspectives de recettes fiscales (Barr, 1993), de productivité de l'ensemble des salariés (Moretti, 2004) ou encore de dynamisme démocratique (Dee, 2004 ; Haveman et Wolfe, 1984 ; Milligan et al., 2004). 
De même, d'autres travaux s'efforcent de justifier et de construire les conditions d'une mise en œuvre de frais d'inscription dans le cas français : Grégoir (2008) et Courtioux (2009 ; 2010) soulignent l'importance des rendements privés de l'éducation et la légitimité qui résulterait, en termes d'équité, de l'instauration d'une contribution des étudiants. S’inspirant de modèles étrangers, ils définissent alors les conditions d'accompagnement nécessaires à un rehaussement des frais d'inscription et appellent notamment à la mise en place de PARC (Prêts À Remboursement Conditionnel). Dans la même veine, un numéro spécial de la Revue d'économie politique, dirigé par d'Autume et al. (2006), fait référence aux besoins financiers de l'université française et propose des arrangements institutionnels permettant de faire contribuer davantage les étudiants aux coûts de leurs études. Ces arguments théoriques sont repris, par exemple, dans un rapport du CGEFI (2015) recommandant la hausse des frais d'inscription des écoles d'ingénieur au motif des importants rendements privés de ces études : "La hausse des tarifs sur trois ans correspond à un peu plus de deux mois de salaire de première embauche."

Flacher et $a l$. (2013) répondent à ces différents arguments. Ils montrent que pour que l'introduction de frais d'inscription rende l'enseignement supérieur plus équitable, il faudrait que son financement soit réellement progressif et que les compensations financières en direction des étudiants les moins favorisés soient suffisantes, ce qui n’a jamais été le cas dans les différents pays ayant opté pour une introduction ou une augmentation des frais d'inscription.

Il existe par ailleurs un risque de polarisation du système universitaire lorsque les frais d'inscription augmentent (Epple et Romano, 2004 ; Hsieh et Urquiola, 2006), venant mettre à mal la rhétorique sur l'équité des frais d'inscription.

Concernant l'efficience des frais d'inscription, de nombreux résultats de travaux empiriques - concluent, d'une part, à l'existence d'une contrainte d'endettement impactant en priorité les étudiants issus des catégories sociales les moins favorisées (Callender et Jackson, 2005) et, d'autre part, au fait que la dette distord les comportements des étudiants $^{10}$ (Cameron et Heckman, 2001 ; Field, 2009). Ils vont à l'encontre des travaux théoriques concluant à une prétendue efficience des frais d'inscription.

De la même manière, en étudiant l'introduction des frais d'inscription à l'université Paris Dauphine, Moulin et al. (2014) montrent que celle-ci a eu des effets ségrégatifs et inégalitaires en termes de sélection des trajectoires universitaires conduisant à un recrutement dans cette université, mais qu'elle n'a pas eu d'effet, contrairement aux prescriptions des modèles théoriques, sur la réussite des étudiants.

10. Field (2009) révèle que le poids de la dette étudiante conduit à délaisser les emplois publics en raison de leur rémunération plus faible. Cameron et Heckman (2001) montrent que la dette influence les décisions des étudiants en les incitant à privilégier des formations courtes et professionnalisantes. 
Enfin, concernant la capacité des frais d'inscription à répondre aux besoins de financement de l'enseignement supérieur, les expériences étrangères montrent que l'État se désengage massivement du financement de l'éducation, au profit des financements privés, de telle sorte que l'effet soit quasiment nul sur les budgets totaux.

Ainsi, de 2005 à 2008, au Royaume-Uni, les dépenses de l'État pour les universités sont passées de 14,1 à 11,9 milliards de livres, tandis que les dépenses des ménages augmentaient de 5,8 à 9,5 milliards de livres (Moulin, 2014a). De la même manière, le relèvement du plafond des frais d'inscription, en 2012, s'est accompagné d'une baisse de $40 \%$ des dotations de l'État aux établissements; pour autant, cela ne lui a pas permis de réaliser des économies dans la mesure où il doit désormais supporter l'augmentation du taux de défaut sur les prêts étudiants (Moulin, 2014b).

Aux Etats-Unis, le volume des dettes étudiantes a dépassé, en 2010, celui des prêts automobiles et des cartes de crédits, et s'élève à plus de 1000 milliards de dollars. La littérature discute de la pertinence de ce volume sur la base des rendements individuels du capital humain (Avery et Turner, 2012 ; Dynarski, 2014). En plus de leur volume total, ces prêts inquiètent par leur similarité avec les mécanismes de subprimes: les agences privées qui accordent les prêts aux étudiants sont assurées contre les pertes en cas de défaut, non par une titrisation comme pour les subprimes, mais par l'Etat fédéral. Elles sont donc incitées à les attribuer très largement, sans limitation sur la qualité de l'enseignement suivi en amont. De même, elles font peu d'effort pour étaler les remboursements en cas de difficultés rencontrées par les diplômés, le défaut pur et simple étant pour elles plus rentable.

Face aux limites d'un mode de financement de l'enseignement supérieur reposant sur les frais d'inscription, il est pourtant possible, à l'image de ce qui se pratique dans certains pays du nord de l'Europe (Moulin, 2015), de faire reposer le financement des universités sur la société dans son ensemble et d'octroyer à tous les étudiants une allocation afin qu'ils réalisent leurs études indépendamment de leur origine sociale ${ }^{11}$.

Bien que la mise en place d'un tel système soit nécessairement coûteuse, la contribution d'Aurélien Casta révèle que l'émergence et le maintien d'établissements privés à but lucratif coûtent cher aux finances publiques; il y a peut-être là matière à réflexion collective sur le financement de l'enseignement supérieur et de la recherche.

\section{Conclusion}

Dans l'introduction de ce dossier, Olivia Chambard et Laurène Le Cozanet développent une discussion pertinente sur le risque de réification de l'Université encouru par une analyse en termes d' "économicisation ». Parce que l'enseignement supérieur participe de la société, il a nécessairement des liens avec le monde économique

11. C'est notamment ce que proposent Flacher et al. (2012). 
Il s'est néanmoins distingué, à différentes époques, par une revendication d'autonomie (Rikap, 2015), vis-à-vis du pouvoir mais aussi des intérêts économiques.

La création de l'Université de Paris, en 1215 (Verger, 1997, p. 70) comme le modèle de Humbolt (Von) (1809 [1979]) confèrent un rôle central à cette autonomie. Cette singularité de l'Université, même fantasmée, n'en est pas moins opérante dans l'investissement de certains étudiants, guidés par une vocation ou une curiosité intellectuelle et dans la mobilisation d'une partie au moins des universitaires.

Vatin et al. (2010, p. 172) interprètent ainsi la grève des universitaires de 2009 comme la manifestation d'un attachement à " un type de gestion de l'Université sur le mode d'un ordre professionnel, soudé par la vertu supposée de ses membres, d'un corps régi par une norme antiutilitariste ". On retrouve le même type d'appel aux valeurs de l'Université chez des enseignants de SciencesPo, choqués par la répartition d'importants bonus entre les membres de la direction de l'institut, qui "nous semble contraire à l'esprit du service public et à l'éthique de l'enseignement et de la recherche, mais aussi parce qu'elle est totalement déconnectée du monde universitaire auquel l'établissement prétend appartenir $»^{12}$. La négation symbolique autant que matérielle de ces valeurs participe d'ailleurs de l'augmentation de la souffrance au travail chez les universitaires (Gaulejac (De), 2012, pp. 17-18).

Ainsi, depuis les années 2000, les liens avec le monde économique prennent une nouvelle tournure (Granger, 2015), vers une mise en marché.

La généralisation des frais d'inscription significatifs confere au renforcement de la concurrence une tournure pour partie - si ce n'est principalement - économique à l'enseignement supérieur et à la recherche.

Les fonds ainsi drainés matérialisent, sous la forme d'un capital économique, le capital académique des établissements les mieux évalués, avec le risque d'un renforcement de la polarisation des systèmes d'enseignement supérieur.

Le cas de la France révèle que cette polarisation n'est pas nouvelle (avec la dualité universités/grandes écoles), mais tout indique qu'elle ne fait que s'accroître à partir des premières expériences d'augmentation des frais et de concentration des établissements prétendant au statut « d'universités de classe mondiale ».

Sans réduire la complexité des phénomènes ou nier le maintien de spécificités qui distinguent l'enseignement supérieur du reste du monde économique, on peut soutenir qu'il s'organise de moins en moins en termes de service public, de droit à l'enseignement et de vocation et davantage en termes de concurrence, d'investissement en capital humain et d'intérêt économique.

12. Voir l'article intitulé "Sciences-Po : "Des enseignants exigent des explications de Richard Descoings ", publié par une ou des personnes ayant gardé l'anonymat sur le site Médiapart, le 20 février 2012. 


\section{- Bibliographie}

Acides (2015), Arrêtons les frais! Pour un enseignement supérieur gratuit et émancipateur, Paris, Raisons d'agir.

Aghion P. and Howitt P. (2009), The Economics of Growth, Cambridge, MIT Press.

Avery Ch. and Turner S. (2012), "Student Loans: Do College Students Borrow Too Much-Or Not Enough?” Journal of Economic Perspectives 26(1), pp. 165-92.

Autume (d') A., Le Merrer P. et Pisani-Ferry J. (2006), "Financement et organisation de l'enseignement supérieur : vers un nouveau modèle? ", Revue d'économie politique 116(6), pp. 743-744.

Barats C. et Leblanc J.-M. (2013) «Généalogie de la co-construction médiatique du "classement de Shanghai" En France. Corpus de Presse et Usages Sociodiscursifs ", Mots. Les langages du politique 102(2), pp. 67-83.

Barr N. (1993), "Alternative Funding Resources for Higher Education", The Economic Journal, 103(418), pp. 718-728.

Becker G. (1962), «Investment in Human Capital: A Theoretical Analysis - Part 2 », The Journal of Political Economy 70(5), pp. 9-49.

Callender C. et Jackson J. (2005), "Does the Fear of Debt Deter Students from Higher Education ?", Journal of Social Policy 34(4), pp. 509-540.

Cameron S. et Heckman J. (2001), "The Dynamics of Educational Attainment for Black, Hispanic, and White Males", Journal of Political Economy 109(3), pp. 455-499.

Card D. (1999), "The Causal Effect of Education on Earnings", in Handbook of Labor Economics, by Ashenfelter O. and Card D, North Holland, Elsevier, pp. 1801-1863.

Charles N. et Delpech Q. (2015), Investir dans l'internationalisation de l'enseignement supérieur, France Stratégie.

Cgefi (2015), " Revue des dépenses des écoles publiques d’ingénieurs », Contrôle général économique et financier.

Chiappori P.-A. (2011), Financement de l'enseignement supérieur : quelle place pour les entreprises?, Institut de l'entreprise.

Colin N. (2008), Pour une contribution plus juste au systéme d'enseignement supérieur, Institut Montaigne.

Cour Des Comptes (2012), Sciences Po : Une forte ambition, une gestion défaillante, Rapport de la cour des comptes.

Courtioux P. (2009), « Peut-on financer l'éducation du supérieur de manière plus équitable? ", Position Papers EDHEC. 
Courtioux P. (2010), «L'effet du système socio-fiscal sur les rendements privés de l'enseignement supérieur. Une analyse par microsimulation ", Position Papers EDHEC.

Dee T. (2004), “Are there Civic Returns to Education?”, Journal of Public Economics 88(910), pp. 1697-1720.

Dynarski S. (2014), “An Economist's Perspective on Student Loans in the United States", ES Working Papers Series.

Epple D. and Romano R. (1998), "Competition Between Private and Public Schools, Vouchers, and Peer-Group Effects”, American Economic Review 88(1), pp. 33-62.

Espeland W. and Sauder M. (2007), "Rankings and Reactivity: How Public Measures Recreate Social Worlds”, American Journal of Sociology 113(1), pp. 1-40.

Field E. (2009), "Educational Debt Burden and Career Choice : Evidence from a Financial Aid Experiment at NYU Law School”, American Economic Journal: Applied Economics 1(1), pp. 1-21.

Flacher D., Harari-Kermadec H. et Moulin L. (2013a), "Faut-il (vraiment) augmenter les frais d'inscription à l'université ? ", Revue française d'économie 27(3), pp. 145-183.

Flacher D., Harari-Kermadec H. et Moulin L. (2013b), « Régime par répartition dans l'enseignement supérieur : fondements théoriques et estimations empiriques ", Working paper du CEPN 2012-06.

Garces E., Thomas D. and Currie J. (2002), "Longer-Term Effects of Head Start", American Economic Review 92(4), pp. 999-1012.

Gardner M. (2008), “A Top 10 University System for Australia”, RMIT Vice Chancellor’s speech.

Gary-Bobo R. Et Trannoy A. (2005), « Faut-il augmenter les droits d'inscription à l'université ? ", Revue française d'économie 19(3), pp. 189-237.

Gary-Bobo R. and Trannoy A. (2008), "Efficient Tuition Fees and Examinations”, Journal of the European Economic Association 6(6), pp. 1211-1243.

Gaulejac (de) V. (2012), La recherche malade du management, Versailles, Quae.

Gazier B. (1992), Économie du travail et de l'emploi, Paris, Dalloz.

Gingras Y. (2013), Les dérives de l'évaluation de la recherche : du bon usage de la bibliométrie, Paris, Raisons d'agir.

Granger C. (2015), La destruction de l'université française, Paris, La Fabrique.

Grégoir S. (2008), "Les prêts étudiants peuvent-ils être un outil de progrès social ?", Position Papers, EDHEC. 
Grossman M. (2006), "Education and Nonmarket Outcomes", in Handbook of the Economics of Education, by Hanushek E. et Welch F., North Holland, Elsevier, pp. 577-633.

Grossman M. and Kaestner R. (1997), "Effects of Education on Health", in The Social Benefits of Education, by Behrman J. et Stacey N., Michigan, University of Michigan Press, pp. 9-123.

Harmon C., Oosterbeek H. and Walker I. (2003), “The Returns to Education: Microeconomics", Journal of Economic Surveys 17(2), pp. 115-156.

Hautier A., Colas F., D’harcourt J., Rutenburg S., Toussain R., Aimé P., Kretchner A., Pimmel É., Saguet M. (2015), "La situation financière des universités », Inspections générales des finances et de l'administration de l'éducation nationale et de la recherche.

Haveman R. and Wolfe B. (1995), “The Determinants of Children's Attainments: A Review of Methods and Findings", Journal of Economic Literature 33(4), pp. 1829-1878.

Hazelkorn E. (2015), Rankings and the reshaping of higher education: The battle for worldclass excellence, London / New York, Palgrave Macmillan.

Hendricks L. (2004), A Database of Mincerian Earnings Regressions, Page personnelle de L. Hendricks.

Hsieh C.-T. and Urquiola M. (2006), "The Effects of Generalized School Choice on Achievement and Stratification: Evidence from Chile's Voucher Program”, Journal of Public Economics 90(8-9), pp. 1477-1503.

Humboldt (von) W. (1809(1979)), «Sur L'organisation Interne et Externe Des établissements Scientifiques Supérieurs à Berlin ", in Philosophies de l'université: l'idéalisme allemand et la question de l'Université, by Ferry L, Pesron J.-P. et Renaut A, Paris, Payot, pp. 319-329.

INSEE (2012), «La répartition des dépenses publiques de l'enseignement supérieur et des aides associées ", Note n 2497/DG75-F120/

Jongbloed B. (2003), "Marketisation in Higher Education, Clark's Triangle and the Essential Ingredients of Markets", Higher Education Quarterly 57(2), pp. 110-135.

Lichtenberger Y. et Aïdara A. (2011), "Faire réussir nos étudiants, faire progresser la France : propositions pour un sursaut vers la société de la connaissance ", Projet 2012 12, Terra Nova.

Lochner L. and Moretti E. (2004), “The Effect of Education on Crime: Evidence from Prison Inmates, Arrests, and Self-Reports", American Economic Review 94(1), pp. 155-189.

MESR (2015), L'état de l'enseignement supérieur et de la recherche en France, $\mathrm{n}^{\circ} 8$, ministère de l'Enseignement supérieur et de la Recherche. 
Milligan K., Moretti E. and Oreopoulos P. (2004), "Does Education Improve Citizenship? Evidence from the United States and the United Kingdom”, Journal of Public Economics 88(9-10), pp. 1667-1695.

Moretti E. (2004), “Workers' Education, Spillovers, and Productivity: Evidence from Plant-Level Production Functions”, American Economic Review 94(3), pp. 656-690.

Moulin L. (2014a), Frais d'inscription dans l'enseignement supérieur : enjeux, limites et perspectives, Thèse de doctorat, université Paris 13.

Moulin L. (2014b), «Une hausse des frais d'inscription en France est-elle inéluctable ou même simplement souhaitable?", Savoir/Agir 29, pp. 43-50.

Moulin L. (2015), « Frais d'inscription dans l'enseignement supérieur et régimes d'Étatsprovidences : une analyse comparative ", Éducation et sociétés 36(2), à paraître.

Moulin L., Flacher D. et Harari-Kermadec H. (2014), "Tuition Fees and Social Segregation: Lessons from a Natural Experiment at the University of Paris 9-Dauphine”, document de travail, CEPN, 2014-18.

OCDE (2013a), "Quels facteurs influencent la mobilité internationale des étudiants ?", Indicateurs de l'éducation à la loupe 5.

OCDE (2013b), Regards sur l'éducation.

Rikap C. (2015), « De la spécificité de l’Université dans le développement d'une stratégie de transformation ", Contretemps.eu.

Rotschild M. and White L. (1995), “The Analytics of the Pricing of Higher Education and Other Services in Which the Customers Are Inputs", Journal of Political Economy 103(3), pp. 573-586.

Teixeira P., Jongbloed B., Dill, D. and Amaral A. (2004), Markets in Higher Education. Rhetoric or Reality?, Dordrecht / Boston / London, Kluwer Academic Publishers.

Vatin F., Caillé A. et Favereau O. (2010), «Réflexions croisées sur la mesure et l'incertitude ", Revue française de gestion, 203(4), pp. 163-181.

Verger J. (1997), "L'essor des universités au XIII siècle », Paris, Les éditions du Cerf. 